\title{
Magnetic field annihilation and reconnection driven by femtosecond lasers in inhomogeneous plasma
}

\author{
Wang YouYuan ${ }^{1,2}$, Li FeiYu ${ }^{3}$, Chen Min ${ }^{1,2}$, Weng SuMing ${ }^{1,2}$, Lu QuanMing ${ }^{4}$, Dong QuanLi ${ }^{2,5}$, \\ Sheng ZhengMing ${ }^{1,2,3^{*}}$, Zhang $\mathrm{Jie}^{1,2}$ \\ ${ }^{1}$ Key Laboratory for Laser Plasmas (Ministry of Education) and School of Physics and \\ Astronomy, Shanghai Jiao Tong University, Shanghai, 200240, China \\ ${ }^{2}$ Collaborative Innovation Center of IFSA (CICIFSA), Shanghai Jiao Tong University, Shanghai \\ 200240, China \\ ${ }^{3}$ SUPA, Department of Physics, University of Strathclyde, Glasgow G4 ONG, UK \\ ${ }^{4}$ CAS Key Lab of Geospace Environment, Department of Geophysics and Planetary \\ Science, University of Science and Technology of China, Hefei 230026, China \\ ${ }^{5}$ School of Physics and Optoelectronic Engineering, Ludong University, Yantai 264025, China \\ *Email: zmsheng@sjtu.edu.cn
}

\begin{abstract}
:
The process of fast magnetic reconnection driven by intense ultra-short laser pulses in underdense plasma is investigated by particle-in-cell simulations. In the wakefield of such laser pulses, quasi-static magnetic fields at a few mega-Gauss are generated due to nonvanishing cross product $\nabla(n / \gamma) \times \vec{p}$. Excited in an inhomogeneous plasma of decreasing density, the quasi-static magnetic field structure is shown to drift quickly both in lateral and longitudinal directions. When two parallel-propagating laser pulses with close focal spot separation are used, such field drifts can develop into magnetic reconnection (annihilation) in their overlapping region, resulting in the conversion of magnetic energy to kinetic energy of particles. The reconnection rate is found to be much higher than the value obtained in Hall magnetic reconnection model. Our work proposes a potential way to study magnetic reconnection related physics with short-pulse lasers of terawatt peak power only.
\end{abstract}

Keywords: magnetic reconnection, laser wakefield, magnetic field generation

PACS number(s): 47.75.+f, 52.35.-g, 52.65.-y, 98.70.Sa 


\section{Introduction}

Magnetic reconnection (MR) is a process by which oppositely directed magnetic field lines squeeze each other and reconnect, converting magnetic energy into particles' kinetic energy [1-3]. It is widely believed to play a key role in many explosive plasma phenomena, such as solar flares, fusion plasma instabilities, and laser-driven plasma jets [4-7]. In the past decade, there has been increasing interest to study MR related processes in laboratory by use of high power laser produced plasma [2,7-8] and other plasma devices [9]. For MR in laser produced plasma, most experimental and numerical investigations have been carried out with nanosecond kJ-level high energy lasers $[6-8,10]$, where the magnetic fields are produced via the Biermann battery process due to nonparallel temperature and density gradients [11-12]. The involved magnetic fields are normally located near the target surface and typically at the mega-gauss (MG) level. Significant progress has been made in this field [13]. For example, three well-collimated high-speed electron jets were observed in the fanlike outflow region in experiments [6,14], which are consistent with the signatures of MR simulations [15]. Fully kinetic particle simulations showed that fast reconnection in these strongly driven systems can be explained by magnetic flux pileup at the shoulder of the current sheet [16]. However, most of the simulations did not include the laser-plasma interaction stages, by simply assuming that two bubbles with high energy electron currents start to evolve in a background plasma.

With the development of ultrashort high power laser technologies, petawatt lasers with picosecond to femtosecond durations are available nowadays. This makes it possible to investigate fast magnetic field generation and dissipation under even higher quasi-static magnetic fields over $100 \mathrm{MG}$ by the relativistic intense lasers interaction with plasmas $[2,16-18]$ or by the interaction of high current relativistic electron beams with plasmas $[19,20]$.

In this paper, we report particle-in-cell (PIC) simulations of the MR process driven by intense femtosecond laser in inhomogeneous plasma. The idea is to send two parallel-propagating femtosecond laser pulses into a nonuniform layer of underdense plasma. High quasi-static magnetic fields are generated in the wakefield behind the pulses due to nonvanishing cross product [21] $\nabla(n / \gamma) \times \vec{p}$, where $n$ is the electron density, $\vec{p}$ is the 
electron fluid momentum and $\gamma$ is the corresponding relativistic factor, respectively. The down-ramp density inhomogeneity causes lateral drift of the generated magnetic fields [22-23], and as a result, reconnection occurs when the magnetic fields having different polarities drift toward each other and become partially overlapped. This process can induce substantial electrostatic fields which accelerate charged particles and cause plasma heating. Prominently, such a setup features an ultra-fast reconnection rate, typically at the time scale of a few hundred femtoseconds.

\section{Quasi-static magnetic field generation and drift}

It is well-known that large-amplitude plasma waves can be excited by an ultra-short intense laser pulse in underdense plasma. The wakefield can be as high as $1 \mathrm{GV} / \mathrm{cm}$ and move with a phase velocity close to the group velocity of the laser pulse, making it ideal for compact particle acceleration [24-25]. In addition, quasi-static magnetic fields are produced due to wakefield excitation. Using the term "quasi-static", we mean the magnetic fields evolve with a time scale much longer than the laser oscillation period. The equation for magnetic field generation in the laser wakefield can be described by the general equation [21]:

$$
\left(\nabla^{2}-\frac{1}{c^{2}} \frac{\partial^{2}}{\partial t^{2}}-\frac{n}{\gamma} \frac{\omega_{p}^{2}}{c^{2}}\right) \vec{B}=\frac{\omega_{p}}{c} \nabla\left(\frac{n}{\gamma}\right) \times \vec{p},
$$

where $\omega_{p}^{2}=n_{0} e^{2} / \epsilon_{0} m_{e}$, the magnetic field $|\vec{B}|$ is normalized by $m_{e} \omega_{p} / e$, the density $\mathrm{n}$ is normalized by $n_{0}$ (the initial plasma density), the electron fluid momentum $|\vec{p}|$ is normalized by $m_{e} c$, and $\gamma$ is the relativistic factor of the electron fluid. The nonvanishing cross product or the source term on the right-hand side of Eq. (1) $\vec{S}=\nabla(n / \gamma) \times \vec{p}$ is responsible for the magnetic field generation. In homogeneous underdense plasma, Eq. (1) can be simplified to

$$
\left(\nabla_{\perp}^{2}-\frac{n}{\gamma} \frac{\omega_{p}^{2}}{c^{2}}\right) \vec{B}=\frac{\omega_{p}}{c} \nabla\left(\frac{n}{\gamma}\right) \times \vec{p} .
$$

The source term points to the azimuthal direction in three-dimensional (3D) geometry as the laser pulse drives a net electric current to the opposite of the laser propagation direction. The scaling of the magnetic field strength with the driving laser intensity obtained as $|\vec{B}| \propto I_{L}^{2}$ under weakly relativistic laser intensity $I_{L}$ and $|\vec{B}| \propto I_{L}$ for highly relativistic laser intensity [21]. It has also been shown that the magnetic field profile produced in 
homogeneous plasmas changes little up to the time scale of ion motion. Fast evolutions on the scale of electron motion are, however, anticipated in inhomogeneous plasmas by several theory models $[22,23]$. In particular, being excited along a down-ramp density profile, the magnetic field tends to rapidly drift along transverse and longitudinal directions. In the following, we carry out particle-in-cell (PIC) simulations with the code OSIRIS [26], to study the field evolutions and identify possible consequences of magnetic reconnection when two parallel-propagating laser pulses with close focal spot separations are sent to the plasma.

We begin with 2D PIC simulations, which although described in a simplified slab geometry, can capture the key features of the present problem and can provide a reference for further 3D simulation shown later. Figure $1(\mathrm{a})$ shows the simulation box, which is $115 \mu \mathrm{m}$ and $60 \mu \mathrm{m}$ long in the $\mathrm{X}$ and $\mathrm{Y}$ directions, respectively. An underdense plasma consisting of electrons and protons is initialized in $0<X<85 \mu \mathrm{m}$. While the transverse density is uniform, the longitudinal density profile is given by the green curve in Fig. 1(a), i.e., $\mathrm{n}_{0}=0.01 \mathrm{n}_{\mathrm{c}}$ in $0<X<30 \mu \mathrm{m}$, then decreases as $\mathrm{n}=\mathrm{n}_{0}\left[1-\sin \left(\frac{\pi(\mathrm{x}-30)}{100}\right)\right]$ in $30 \mu \mathrm{m}<X<70 \mu \mathrm{m}$, and then remains constant again in $70 \mu \mathrm{m}<X<85 \mu \mathrm{m}$, where $\mathrm{n}_{\mathrm{c}}$ is the critical density. A linearly polarized laser pulse polarized along the $Z$ direction and with the wavelength $\lambda_{L}=1.0 \mu \mathrm{m}$ is incident along the $\mathrm{X}$ direction from the left boundary. It has a focal spot radius $w_{0}=4.0 \mu \mathrm{m}$ and pulse duration $\tau=13 T_{0}=40 \mathrm{fs}$, where $T_{0}=\lambda_{L} / c \approx$ 3.3fs. The normalized laser electric field is $a_{0}=\mathrm{e} E_{0} / m_{e} \omega_{0} c=1.0$, where $E_{0}$ is the peak amplitude laser electric field, $\omega_{0}$ is the laser frequency. It is related to the laser intensity by $I_{0}=1.37 \times 10^{18} \mathrm{~W} / \mathrm{cm}^{2}\left(\mu \mathrm{m} / \lambda_{L}\right)^{2} a_{0}^{2}$. Absorbing boundary conditions are used for both particles and fields. Note that compared with the work by Ping et al. [2], much lower laser intensity and plasma density have been used here. The mechanisms for magnetic field generation are completely different for the two cases. 

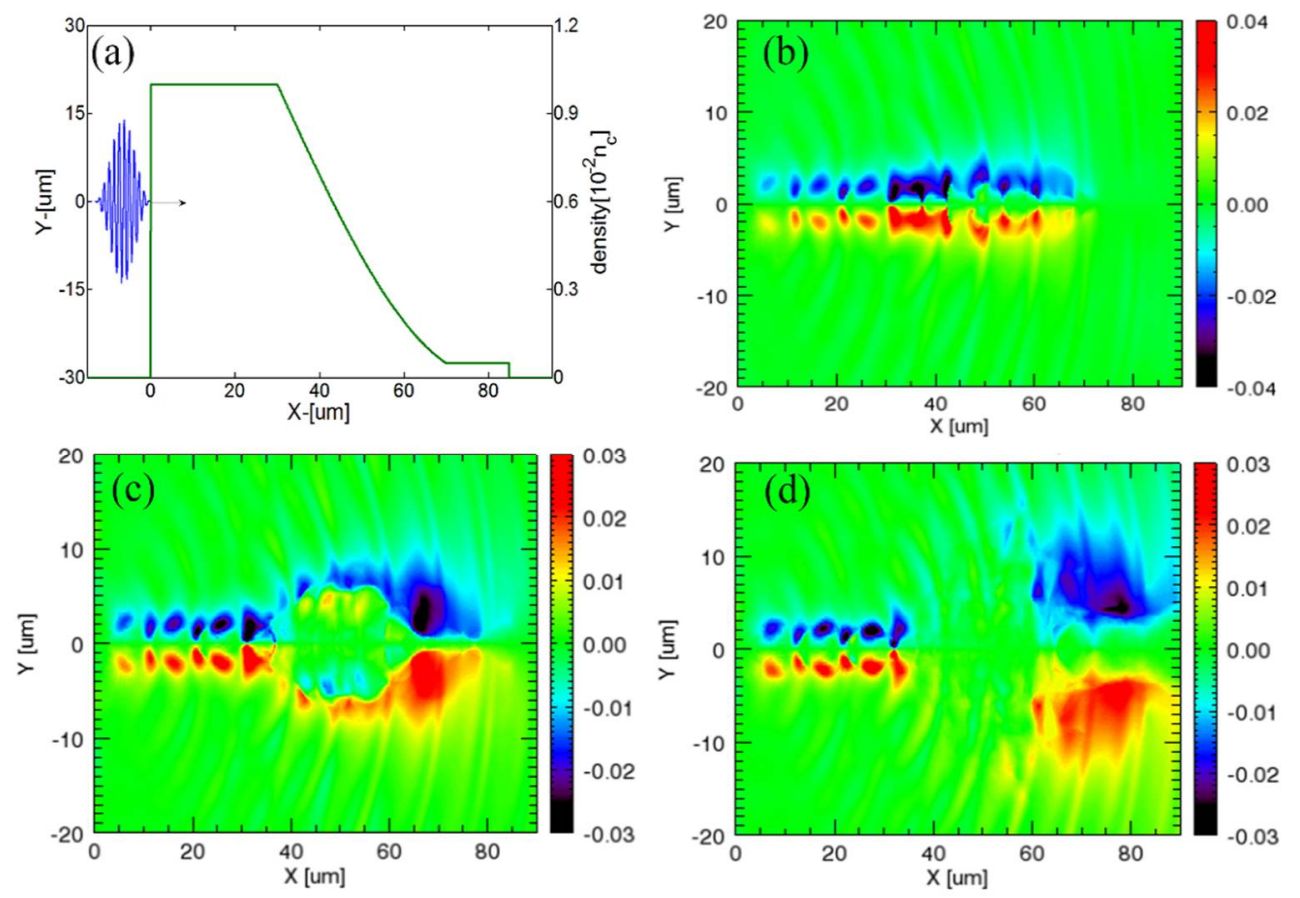

Fig. 1. 2D simulation results of the magnetic fields in the wake region. (a) Initial plasma density distribution and the laser profile along $X$ direction. (b)-(d) The Z-component of the magnetic field $\left(B_{z}\right)$ distribution at $108 T_{0}, 128 T_{0}$ and $160 T_{0}$, respectively. In this and following figures, the magnetic field and electric field are normalized by $B_{N}=m_{e} \omega_{0} / 2 \pi e=1.7 \times 10^{3} T$ and $E_{N}=m_{e} \omega_{0} c / 2 \pi e=$ $5.1 \times 10^{11} \mathrm{~V} / \mathrm{m}$, respectively.

Large-amplitude plasma waves are excited as an intense laser pulse propagates in underdense plasmas, due to the laser ponderomotive force both in the forward and side directions. With the resulting charge separation, the electrons oscillate around their equilibriums with a characteristic frequency roughly given by the electron plasma frequency. The source term of the quasi-static magnetic field on the right-hand side of Eq. (2) points only along the $\mathrm{Z}$ direction in this $2 \mathrm{D}$ slab geometry. Figures $1(\mathrm{~b}-\mathrm{d})$ show snapshots of the magnetic field component along this direction at different stages. In the homogeneous plasma region $(0<X<30 \mu m)$, the magnetic field stays steady with time. It shows an oscillating component around $2 k_{p}$ along the longitudinal direction, in addition to a uniform component which corresponds to a steady electric current driven by the laser pulse [21]. Here $k_{p}$ is the wave number of the plasma wave. The maximum strength of the magnetic field is about $0.05 B_{N}$ or $1 \mathrm{MG}$ under the given laser and plasma conditions. 
In the plasma down-ramp region, however, rapid evolution of $B_{z}$ is found, as also presented in Figs. 1(b)-1(d). The magnetic field structure drifts rapidly in the lateral directions, in addition to longitudinally against the density gradient. The lateral drift is due to the force acting on the magnetic dipole vortex in the $B \times \nabla n$ direction according to Refs. $[22,23]$. Note that this drift motion is completely different from the well-known diamagnetic drift [27]. Here the electron magnetohydrodynamic fluid model is sufficient since the ion motion is negligible in this short period of time [28-29]. Interestingly, there are also opposite magnetic fields generated near the laser axis in the down-ramp as show in Fig. 1(c). Up to $\mathrm{t}=160 T_{0}$, the drift is almost complete and the magnetic field fully gathers in the low density plateau region with the peak spot at around $x=78 \mu \mathrm{m}$, where the initial magnetic fields right after laser passage are weak.

\section{Magnetic field annihilation}

Now we use two laser pulses, each with the same parameters as defined above, and they copropagate but are separated along the $\mathrm{Y}$ direction by $16 \mu \mathrm{m}$, with the spot centers being at $Y= \pm 8 \mu m$, respectively.

The evolution of the magnetic fields produced by the two laser pulses is show in Figs. 2(a), 2(c) and 2(e). Similarly, each pulse generates magnetic fields in their own wakes. Mirrored by the center axis of the box, the magnetic fields show opposite polarities which will drift toward each other around the center in the plasma down-ramp. At $t=108 T_{0}$, the magnetic fields in the low plateau is still negligible and the fields in the down-ramp have not much drifted yet, although the laser pulses have already propagated out of the box. Only $20 T_{0}$ later, the magnetic fields become largely accumulated on the right between $\mathrm{X}=60$ and $80 \mu \mathrm{m}$, with significant transverse expansion. They start to overlap with each other and annihilate near the central axis $(Y=0)$. Full annihilation is achieved in another $30 T_{0}$ up to $\mathrm{t}=$ $160 T_{0}$, as show in Fig. 2(e), while the outer fields have already drifted close to the box boundaries. Importantly, the annihilation of the magnetic fields results in the formation of inductive longitudinal electric fields near the central axis, as progressively depicted in Figs. 2(b), 2(d) and 2(f). At the beginning right after the passage of the driving lasers [Fig. 2(b)], the longitudinal electric fields corresponding to charge separation are dominantly more intense in the high density regions, which is in line with the scaling of the wakefield strength with the plasma density, $E_{x} \propto n^{1 / 2}$. However, as the magnetic field drift takes place, the 
electric fields associated with the laser wakefield excitation weaken rapidly in the inhomogeneous region. At the same time, additional longitudinal electric fields around the central axis arise, which is a solid evidence of the magnetic field annihilation.
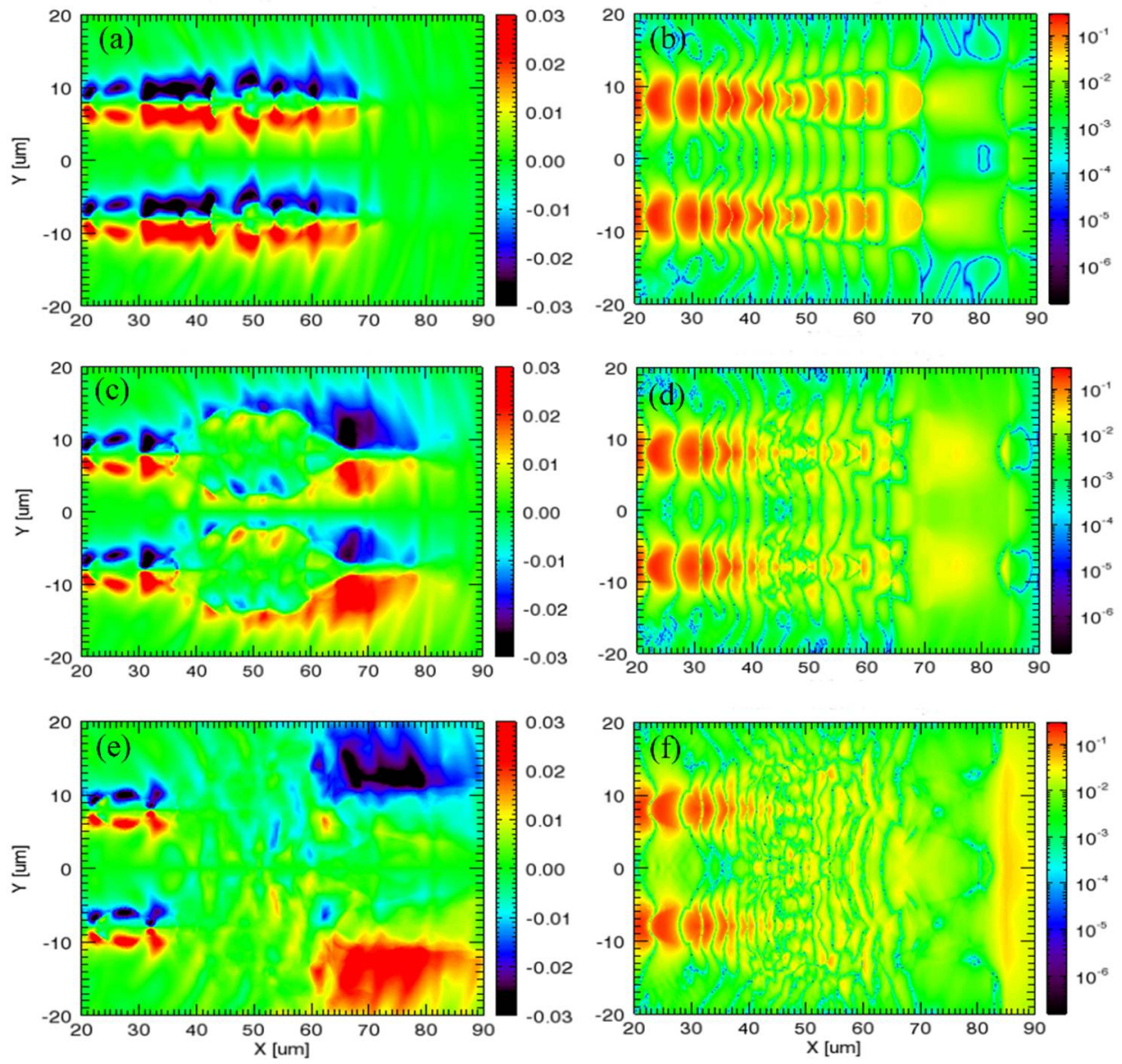

Fig. 2. 2D simulation results with two laser pulses. Plots (a), (c) and (e) show the distributions of the Z-component of the magnetic field $\left(\mathrm{B}_{\mathrm{z}}\right)$ at $108 T_{0}, 128 T_{0}$ and $160 T_{0}$, respectively. Plots $(\mathrm{b}),(\mathrm{d}),(\mathrm{f})$ are the corresponding absolute value of electric field in the $X$ direction $\left(\left|E_{X}\right|\right)$ in logarithmic scale. The magnetic and electric fields are normalized as in Fig. 1.

Figure 3(a) further shows the induced longitudinal electric field $E_{x}$ near the magnetic field annihilation region at $\mathrm{Y}=0$ at $\mathrm{t}=108 T_{0} \& 128 T_{0}$. Consistently, $E_{x}$ is mainly found in the nonuniform plasma region at the beginning free of annihilation, and grows to substantial levels on the right around $70<X(\mu m)<85$ at $\mathrm{t}=128 T_{0}$ when the magnetic annihilation is taking place. In addition, the ion skin depth is about $d_{i}=300 \mu m$ and the electron skin depth is about $d_{e}=7 \mu \mathrm{m}$ in the low density plateau region. The electron cyclotron is about $0.03 \omega_{0}$.according to Figs. 1 and 2 . As the magnetic annihilation occurs in very short period of 
time, ion motion does not play a critical role. The averaged inductive electric field is about $0.01 E_{N}$, which suggests is that the magnetic field energy is efficiently converted to the electric field energy. The inductive electric fields generated in the annihilation region are unipolar and can accelerate charged particles. In this way, the magnetic field energy is again converted into the kinetic energy of plasma. In our case, the inductive electric field represented by the $E_{x}$ component is positive according to the generalized Ohm's law $[2,17,30]$, therefore, it accelerates electrons in the negative direction of the $X$-axis.

We present the electron momentum distribution in Figs. 3(b)-3(d) for the three instants described so far. At $\mathrm{t}=108 T_{0}$, most electrons only have small momenta, since there are few electrons trapped and accelerated by the laser wakefield under the given laser and plasma conditions. A few electrons have positive velocities along $X$ direction, which is mainly due to electron acceleration by the laser ponderomotive force. However, at $\mathrm{t}=160 T_{0}$, a large number of electrons are accelerated considerably along the negative $X$ direction. Obviously, they are accelerated by the inductive electric fields pointing along positive $\mathrm{X}$-axis. During the magnetic annihilation, electrons are mainly accelerated by the longitudinal electric fields, even though their transverse momenta are increased as well due to the presence of transverse magnetic and electric fields, as shown in Fig. 4(d).
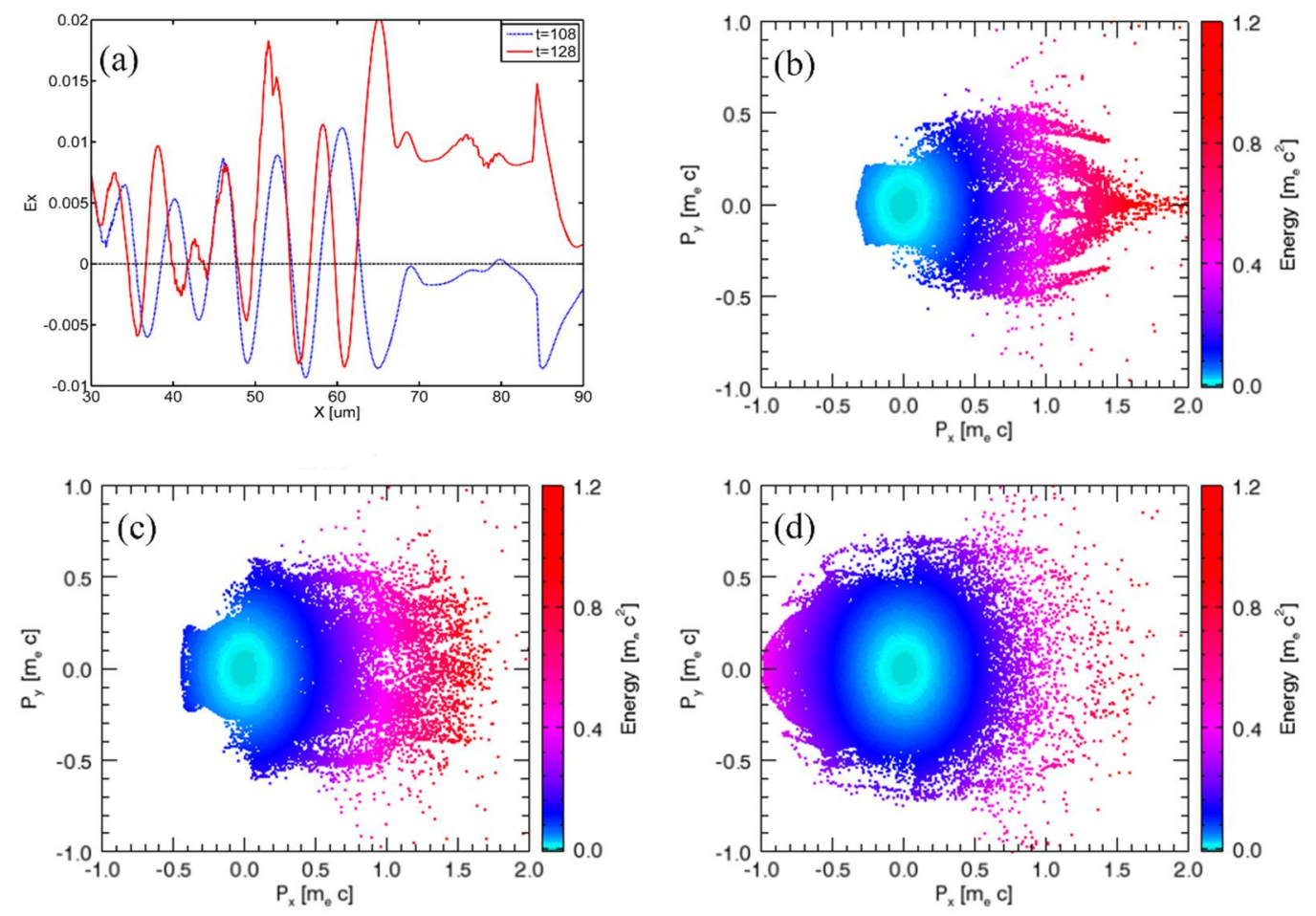

Fig. 3 (a) Longitudinal electric fields $E_{X}$ along the central axis $(Y=0)$ at $108 T_{0}$ and $128 T_{0}$, respectively. Plots (b)-(d) show the electron momentum distributions at $108 T_{0}, 128 T_{0}$ and $160 T_{0}$, respectively, 
where the color bars represent the particle kinetic energy.

\section{Magnetic reconnection}

In order to check 3D effects, we also carry out 3D simulations. The simulation box is $L_{x} \times L_{y} \times L_{z}=115 \times 60 \times 50(\mu \mathrm{m})$ in size, divided into $3450 \times 600 \times 500$ cells. All the laser-plasma conditions are the same as before, with the density being also uniform along the third direction.
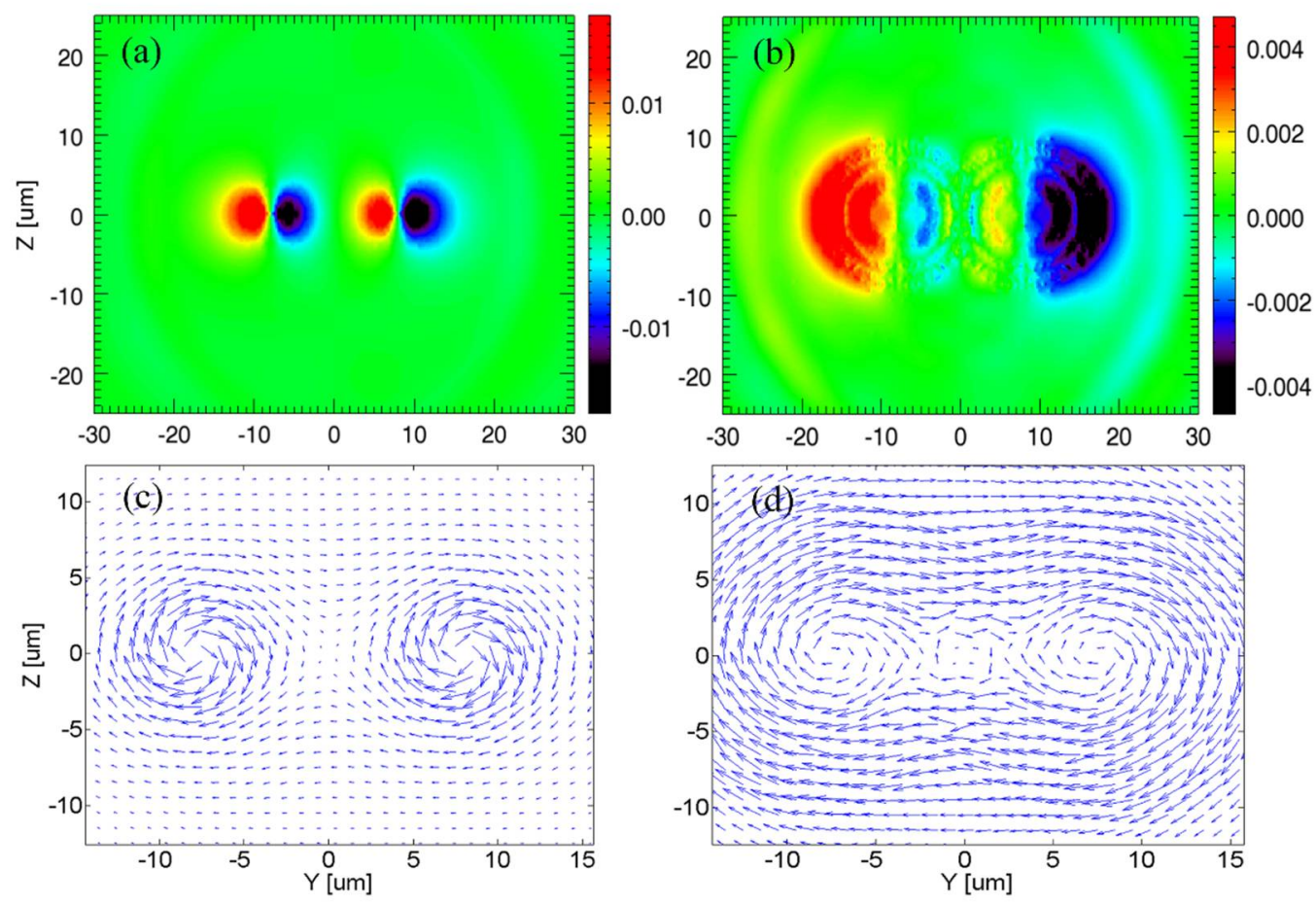

Fig. 4 3D simulation results with two laser pulses. (a) and (b) show the distribution of the Z-component magnetic field $\left(B_{Z}\right)$ in the Y-Z plane at $X=65 \mu \mathrm{m}$ at $108 T_{0}$ and $120 T_{0}$, respectively. Plots (c) and (d) show the corresponding magnetic field vectors $\left(B_{y}, B_{z}\right)$ in the plane at $108 T_{0}$ and $120 T_{0}$, respectively, where the arrows indicate the direction and strength of the magnetic fields.

The most noticeable differences between $3 D$ and 2D simulations are that the self-generated magnetic fields now are azimuthal around the laser axis, in contrast to Z-polarized in the 2D slab geometry. In the density down-ramp region, quasi-static magnetic fields expand radially and magnetic reconnection occurs when the expanding magnetized plasma bubbles meet each other around the box center. This is illustrated in Fig. 4 by transverse cuts of the magnetic fields at $X=65 \mu \mathrm{m}$ for $\mathrm{t}=108 T_{0}$ to $120 T_{0}$. In Figs. $4(\mathrm{a})$ and 4(b), we only present the Z-component of the magnetic fields, $B_{z}$, which have double-lobe 
profiles for each wake. While being azimuthal in nature, the magnetic fields should be annular-like, with the Y-component having similar profiles but in the orthogonal direction. This is more evident in Figs. 4(c) and 4(d) in terms of vector plots. At $\mathrm{t}=108 T_{0}$, the distance of the center points in the two magnetic dipoles is about $4 \mu \mathrm{m}$, it means the two bubbles have expanded but not yet squeeze each other dramatically. With the field drift continues, the magnetic fields gradually squeeze around the box center and form a reconnection $x$-line at the leading point of tangency (namely at $Y=0$ ) between the bubbles. The two magnetic bubbles are reconnected into one in the outer region at $\mathrm{t}=120 T_{0}$, as shown in Fig. $4(\mathrm{~d})$. When the magnetic fields with different polarities meet at the diffusion region, the field annihilation develops and the field structures are rearranged, deriving electric field perpendicular to the plane.

Figure 5 shows the electric field in the 3D simulation. At $X-Z$ plane $(Y=0)$, magnetic reconnection occurs dramatically and derives out electric field, which will accelerate electrons to high speed. Figures $5(\mathrm{a})$ and 5 (c) present the electric field at $108 T_{0}$. At this moment, some of the magnetic field has already been rearranged and induce positive electric field along $X$ direction. This explains why the peak field value is bigger than valley value. Between $70 \mu \mathrm{m}<\mathrm{X}<85 \mu \mathrm{m}$, the initial density is almost zero and the $E_{\mathrm{X}}$ is along positive $X$ direction which indicates magnetic reconnection dominates the electric field. At $t$ $=120 T_{0}$, the periodicity of $\mathrm{E}_{\mathrm{x}}$ is destroyed and the maximum amplitude is about $0.01 E_{N}$. It is the time when magnetic reconnection occurs most dramatically in the $Y-Z$ plane $(X=65)$. At the same time, the reconnection induced electric fields heat the plasma electrons effectively, converting a portion of the magnetic energy to kinetic energy. These energetic electrons may further be diverted by the Hall effect, leading to hot electron jets in the annihilation plane which will be shown in Fig. 6. 

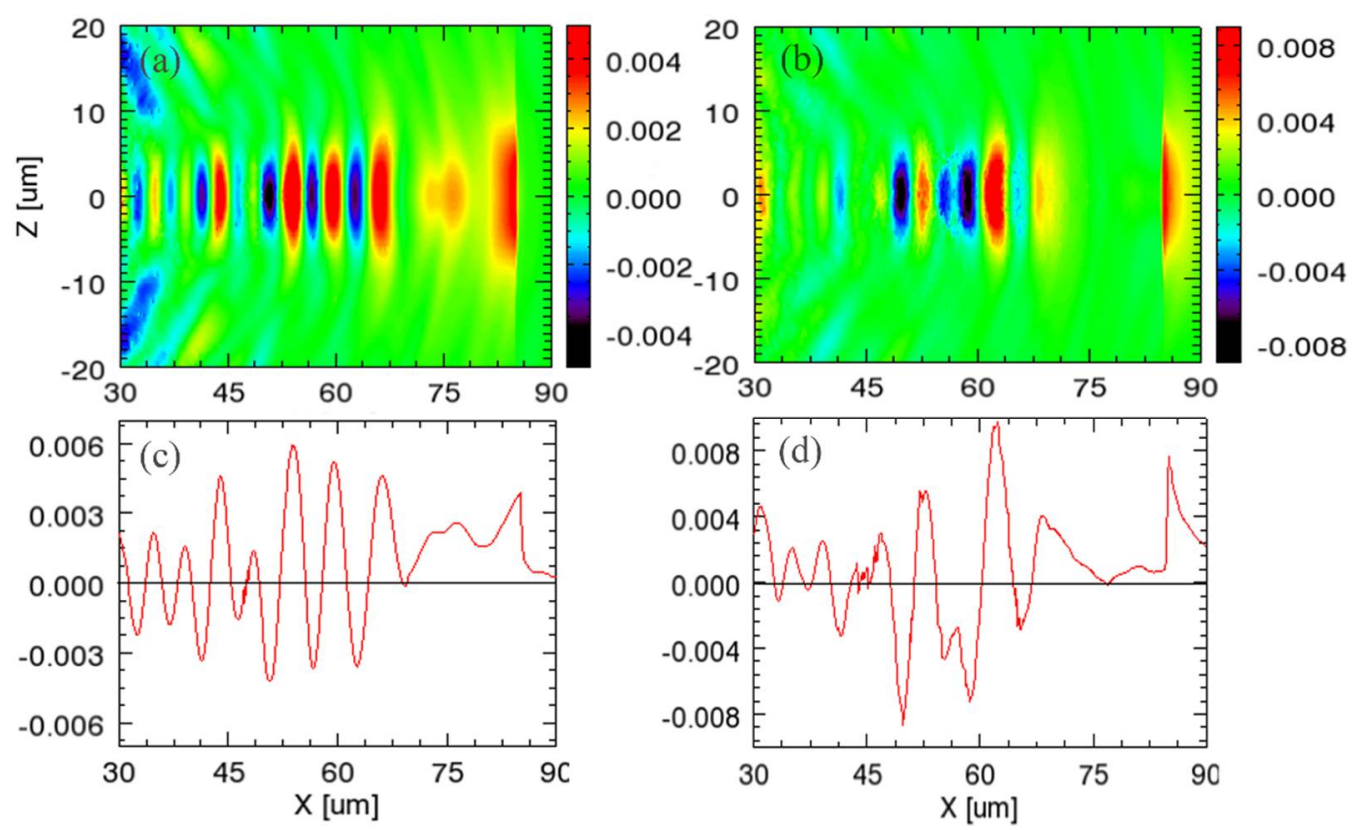

Fig. 5 (a)-(b) The longitudinal electric field $E_{X}$ in the $X-Z$ plane (at $Y=0$ ) at $108 T_{0}$ and $120 T_{0}$. (c)-(d) The corresponding $E_{X}$ along the $X$ axis $(Y=0, Z=0)$.

The momentum space of electrons in $-4 \mu \mathrm{m}<Y<4 \mu \mathrm{m}$ is illustrated in Figs. 6 at $108 T_{0}$ and $160 T_{0}$. At $\mathrm{t}=108 T_{0}$, most of electrons with small momentum gather around zero. After $52 T_{0}$ when the magnetic reconnection is almost completed, the X-component momentum of electrons increased which means the particles are accelerated by the inductive electric field as show in Fig. 6(c). Fig. 6(d) shows both the Y-component and Z-component momentum are increased too. We can see the number of energetic particles increased, which confirms magnetic energy is converted to kinetic energy by the reconnection process. 

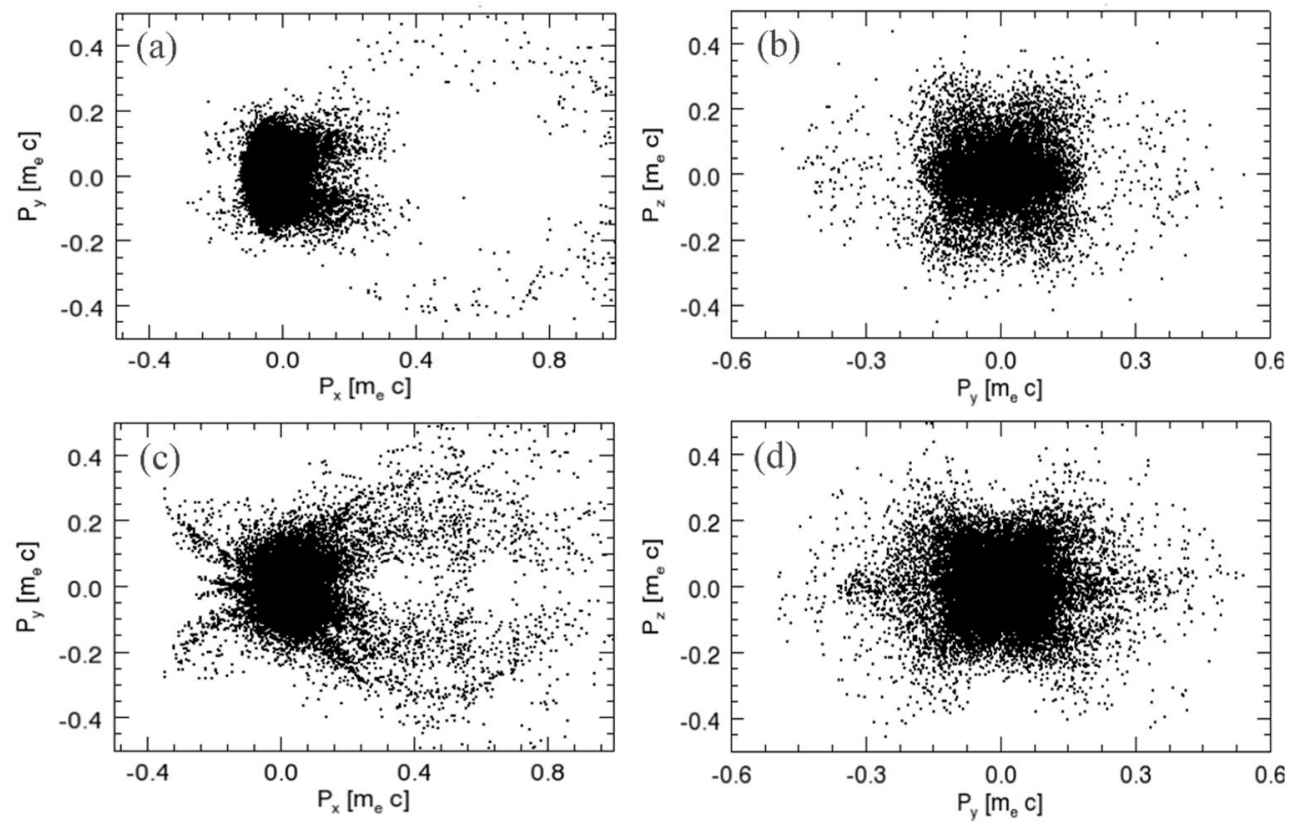

Fig. 6 The momentum space of electrons between $-4 \mu \mathrm{m}<Y<4 \mu \mathrm{m}$. Plots (a) and (c) show electron momentum space $\left(\mathrm{P}_{\mathrm{x}}, \mathrm{P}_{\mathrm{y}}\right)$ at $108 T_{0}$ and $160 T_{0}$. Plots $(\mathrm{b})$ and (d) show electron momentum space $\left(\mathrm{P}_{\mathrm{y}}, \mathrm{P}_{\mathrm{z}}\right)$ at $108 T_{0}$ and $160 T_{0}$.

One of the most important issue in magnetic reconnection process is the reconnection (annihilation) rate $E_{R} / B V_{A}$, where $E_{R}$ is the inductive electric field and $V_{A}$ is the Alfven speed evaluated with plasma density and magnetic field. We calculate the rate in the plateau region $(70 \mu \mathrm{m}<X<85 \mu \mathrm{m})$ where magnetic fields gather together and annihilate. In 2D simulations, the magnetic field $\mathrm{B}=0.03 B_{N}=51 T$, inductive electric field $E_{\mathrm{R}}=0.01 E_{N}=$ $5.1 \times 10^{9} \mathrm{~V} / \mathrm{m}$, plasma density $n=5.658 \times 10^{17} \mathrm{~cm}^{-3}=5 \times 10^{-4} n_{c}$, the corresponding Alfven speed $\mathrm{V}_{\mathrm{A}}=1.48 \times 10^{6} \mathrm{~m} / \mathrm{s}$, about $1 / 200$ of the light speed, reconnection rate amounts to $E_{R} / B_{A}=67.6$. In comparsion, the magnetic field and electric field are $\mathrm{B}=0.015 B_{N}=25.5 T$ and $\mathrm{E}_{\mathrm{R}}=0.002 E_{N}=1.02 \times 10^{9} \mathrm{~V} / \mathrm{m}$ in $3 \mathrm{D}$ simulations. The Alfven speed is estimated to be $V_{A}=7.39 \times 10^{5} \mathrm{~m} / \mathrm{s}$ and reconnection rate is $E_{R} / B_{A}=$ 54.1. Here we have used the density and magnetic field conditions when the magnetic reconnection starts to develop. The rates in both 2D and 3D simulations are much larger than the typical value 0.1 obtained by MHD simulations for Hall magnetic reconnection [31], which mark a prominent feature of this system in underdense plasmas driven by intense short-pulse lasers.

\section{Summary}


To conclude, we have studied magnetic field drift in inhomogeneous plasmas driven by ultrashort intense laser pulses, and consequent magnetic reconnection for two co-propagating pulses with close spot deviations. In the plasma wake behind the laser pulses, high-order electron fluid nonlinearities create intense quasi-static magnetic fields, which drift rapidly in the forward and lateral directions in a density down-ramp plasma. When the magnetic fields with different polarities drift toward each other and annihilate at the diffusion region, electric fields are effectively induced, resulting in significant electron heating. At the same time, hot electron jets in the diffusion region are generated which is one of the signatures to prove the energy conversion from magnetic field to kinetic energy. The reconnection rate found in the simulation is much larger than that the typical value obtained in Hall magnetic reconnection, clear evidence of fast reconnection may due to the strong driven force and Hall effect. Our work suggests a way for experimental realization of fast magnetic reconnection with intense short laser pulses of terawatt peak power only.

\section{Acknowledgements}

This work was partially supported by the National Basic Research Program of China (Grant No. 2013CBA01500), and the National Science Foundation of China (Grant Nos. 11421064 and 11220101002).

\section{References}

1. M. Yamada, Phys. Plasmas 14(5), 058102.(2007)

2. Y. L. Ping, J. Y. Zhong, Z. M. Sheng, X. G. Wang, B. Liu, Y. T. Li, X. Q. Yan, X. T. He, J. Zhang, and G. Zhao, Phys. Rev. E 89(3):031101 (2014)

3. J. Y. Zhong, Y. T. Li, X. G. Wang, J. Q. Wang, Q. L. Dong, C. J. Xiao, S. J. Wang, X. Liu, L. Zhang, L. An, F. L. Wang, J. Q. Zhu, Y. Gu, X. T. He, G. Zhao, J. Zhang, Nat. Phys. 6(12) (2010)

4. E. N. Parker, J. Geophys. Res. 62, 509 (1957).

5. J. B. Taylor, Rev. Mod. Phys. 58(3): 741 (1986)

6. Q. L. Dong, S. J. Wang, Q. M. Lu, C. Huang, D. W. Yuan, X. Liu, X. X. Lin, Y. T. Li, H. G. Wei, J. Y. Zhong, J. R. Shi, S. E. Jiang, Y. K. Ding, B. B. Jiang, K. Du, X. T. He, M. Y. Yu, C. S. Liu, S. Wang, Y. J. Tang, J. Q. Zhu, G. Zhao, Z. M. Sheng, and J. Zhang, Phys. Rev. Lett. 108(1):29-29 (2012)

7. P. M. Nilson, L. Willingale, M. C. Kaluza, C. Kamperidis, S. Minardi, M. S. Wei, P. Fernandes, M. Notley, S. Bandyopadhyay, M. Sherlock, R. J. Kingham, M. Tatarakis, Z. Najmudin, W. Rozmus, R. G. Evans, M. G. Haines, A. E. Dangor, and K. Krushelnick, Phys. Plasmas, 15(9):821 (2008)

8. X. X. Pei, J. Y. Zhong, Y. Sakawa, Z. Zhang, K. Zhang, H. G. Wei, Y. T. Li, Y. F. Li, B. J. Zhu, T. Sano, Y. Hara, S. Kondo, S. Fujioka, G. Y. Liang, F. L. Wang, and G. Zhao, Phys. Plasmas, 23(3):1-23 (2016)

9. R. L. Richard, R. D. Sydora, M. Ashour-Abdalla, Phys. Flu. B Pla. Phys, 2(3) (1990) 
10. W. Fox, A. Bhattacharjee, and K. Germaschewski, Phys. Plasmas, 19(5):603 (2012)

11. C. K. Li, F. H. Séguin, R. P. J. Town, R. D. Petrasso, J. A. Frenje, J. P. Knauer, O. L. Landen, J. R. Rygg, V. A. Smalyuk, Phys. Rev. Lett. 99(5): 055001. (2007)

12. M. G. Haines, Phys. Rev. Lett. 78(2):254-257. (1997)

13. X. G. Wang, C. J. Xiao, Z. Y. Pu, and J. Q. Jia, Chin. Sci. Bull. 57(12): 1369-1374 (2012)

14. K. Huang, C. Huang, Q. L. Dong, Q. M. Lu, S. Lu, Z. M. Sheng, S. Wang, and J. Zhang. Phys. Plasmas, 24, 041406 (2017)

15. S. Lu, Q. M. Lu, C. Huang, Q. L. Dong, J. Q. Zhu, Z. M. Sheng, S. Wang, and J. Zhang, New J. Phys. 16(8):083021 (2014)

16. S. Lu, Q. M. Lu, F. Guo, Z. M. Sheng, H. Y. Wang, and S. Wang, New J. Phys. 2016, 18(1) (2016)

17. Y. J. Gu, O. Klimo, D. Kumar, S. V. Bulanov, T. Z. Esirkepov, S. Weber, and G. Korn, Phys. Plasmas. 22(10), 103113 (2015)

18. Y. J. Gu, Q. Yu, O. Klimo, T. Zh. Esirkepov, S. V. Bulanov, S. Weber and G. Korn, High Power Laser Sci. Eng, 4(2):85-89. (2016)

19. Y. Cui, Z. M. Sheng, Q. M. Lu, Y. T. Li and J. Zhang, Sci China-Phys Mech Astron 58, 105201 (2015)

20. S. Mondal, V. Narayanan, W. J. Ding, A. D. Lad, B. Hao, S. Ahmad, W. M. Wang, Z. M. Sheng, S. Sengupta, P. Kaw, A. Das and G. R. Kumar, Proc. Nat. Acad. Sci. 109, 8011-8015 (2012)

21. Z. M. Sheng, J. Meyer-ter-Vehn, and A. Pukhov, Phys. Plasmas, 5(10): 3764-3773 (1998)

22. S. K. Yadav, A. Das, P. Kaw, Phys. Plasmas, 15(6): 062308 (2008)

23. J. Nycander, M. B. Isichenko, Phys. Fluids B: Plasma Phys. 1990, 2(9): 2042-2047 (1990)

24. T. Tajima, J. M. Dawson, Phys. Rev. Lett. 43(4): 267 (1979)

25. Z. M. Sheng, K. Mima, Y. Sentoku, K. Nishihara, J. Zhang, Phys. Plasmas, 9(7):3147-3153 (2002)

26. R. A. Fonseca, L. O. Silva, F. S. Tsung, V. K. Decyk, W. Lu, C. Ren, W. B. Mori, S. Deng, S. Lee, T. Katsouleas, and J. C. Adam, Computational Science-ICCS 2002, 342-351 (2002)

27. F. F. Chen, Introduction to Plasma Physics and Controlled Fusion, Volume 1: Plasma Physics, 2nd edition, Plenum Press, New York (1984).

28. Q. M. Lu, R. S. Wang, J. L. Xie, C. Huang, S. Lu, and S. Wang, Chin. Sci. Bull 56(12) (2011)

29. J. Guo, Chin. Sci. Bull. 54(3): 456-460 (2009)

30. H. Che, J. F. Drake, M. Swisdak, Nature, 474(7350):184-187 (2011)

31. J. D. Huba, L. Rudakov, Phys. Rev. Lett. 93(17):175003 (2004) 